\title{
Manual educativo de cuidados no pós-operatório de revascularização miocárdica: uma ferramenta para pacientes e familiares
}

\author{
Luana Llagostera Sillano Gentil ${ }^{1}$, Rodrigo Marques da Silva ${ }^{2}$, \\ Sonia Betzabeth Ticona Benavente ${ }^{3}$, Ana Lucia Siqueira Costa ${ }^{4}$
}

\footnotetext{
${ }^{1}$ Enfermeira, Mestre em Enfermagem na Saúde do Adulto. Coordenadora de Enfermagem do Hospital Sírio-Libanês. São Paulo, SP, Brasil. E-mail: luanallgentil@gmail.com.

${ }^{2}$ Enfermeiro, Mestre em Enfermagem. Discente do Programa de Pós-Graduação em Enfermagem na Saúde do Adulto da Escola de Enfermagem da Universidade de São Paulo. São Paulo, SP, Brasil. E-mail: marquessm@usp.br.

${ }^{3}$ Enfermeira, Mestre em Enfermagem na Saúde do Adulto. Discente do Programa de Pós-Graduação em Enfermagem na Saúde do Adulto da Escola de Enfermagem da Universidade de São Paulo. São Paulo, SP, Brasil. E-mail: sonibenavente@usp.br.

${ }^{4}$ Enfermeira, Doutora em Enfermagem na Saúde do Adulto. Professora Doutor da Escola de Enfermagem da Universidade de São Paulo. São Paulo, SP, Brasil. E-mail: anascosta@usp.br.
}

Recebido: 31/08/2016.

Aceito: 18/05/2017.

Publicado: 24/11/2017.

\section{Como citar esse artigo}

Gentil LLS, Silva RM, Benavente SBT, Costa ALS. Manual educativo de cuidados no pós operatório de revascularização miocárdica: uma ferramenta para pacientes e familiares. Rev. Eletr. Enf. [Internet]. 2017 [acesso em:_______19:a38. Disponível em:

http://dx.doi.org/10.5216/ree.v19.43068.

\section{RESUMO}

Elaborou-se um manual educativo para o autocuidado de pacientes revascularizados após a alta hospitalar. Trata-se de um estudo metodológico cuja coleta ocorreu entre fevereiro e abril de 2012 junto a oito peritos em cardiologia e 35 pacientes/familiares. Realizou-se a validação de conteúdo e de face junto a oito pacientes/familiares e oito peritos. Dos 46 itens propostos, os pacientes/familiares classificaram 26 itens (57\%) como muito importante (concordância entre $91,4 \%$ e $100 \%$ ) e os peritos 29 itens (63\%) como muito importante (concordância igual ou superior a 75\%). Selecionaram-se 36 itens distribuídos em 26 categoriais para o manual final. Houve $100 \%$ de concordância de pacientes/familiares e peritos quanto ao conteúdo, linguagem e ilustrações. A ferramenta apresentou validade semântica e adequação de conteúdo para a população, sendo capaz de reduzir complicações decorrentes do despreparo para o autocuidado, reduzindo o número de reinternações e os custos hospitalares, além de sistematizar o ensino pós-operatório.

Descritores: Revascularização Miocárdica; Educação de Pacientes como Assunto; Tecnologia Educacional; Enfermagem.

\section{INTRODUÇÃO}

As doenças cardíacas continuam sendo uma das primeiras causas de morte no mundo atingindo a 17,5 milhões de indivíduos, segundo dados publicados pela Organização Mundial da Saúde ${ }^{(1)}$. Em 2012, o infarto agudo de miocárdio configurouse como a segunda causa de morte, acometendo 6,7 milhões de indivíduos no mundo ${ }^{(1)}$. Além disso, as doenças cardíacas são causas frequentes de internações hospitalares e de elevação de custos nos serviços 
de saúde de países desenvolvidos e em desenvolvimento ${ }^{(2-3)}$. No Brasil, o perfil do paciente com doença cardíaca e as estratégias terapêuticas de atendimento e diagnóstico são similares às utilizadas nos países norte-americanos e europeus. Contudo, a população brasileira demora, em média, 128 minutos mais tempo para procurar os serviços de saúde quando comparada à população dos países desenvolvidos ${ }^{(4)}$. Isto explica, em parte, o pior prognóstico do paciente cardíaco brasileiro na comparação àquele que recebe atendimento precoce, visto que as estratégias de reperfusão miocárdica são tempo-dependentes ${ }^{(5)}$.

O Brasil está à frente da Alemanha, Reino Unido e Japão quanto ao número de cirúrgias cardíacas realizadas - aproximadamente 102.000 em 2012 - sendo prodominante a cirurgia de revascularização miocárdica, das quais $80 \%$ são realizadas no Sistema Único de Saúde(SUS ${ }^{(6)}$. Este procedimento é indicado aos pacientes com dor torácica em repouso, mesmo em uso de outras formas terapêuticas, e com lesões coronarianas extensas que apresentam instabilidade hemodinâmica. Após a alta, as alterações físicas e emocionais são frequentemente relatadas pelos pacientes e se caracterizam com alterações no padrão e qualidade do sono, fadiga, mudança no padrão respiratório, dor torácica, constipação, edema, náuseas, tontura e ansiedade ${ }^{(7-8)}$. Sob essas circunstâncias, o planejamento da alta hospitalar torna-se uma prática essencial de cuidado que contribui para a melhoria dos aspectos mencionados, bem como para maior adesão à terapia medicamentosa e as demais necessidades de cuidado nesta fase de recuperação pós-operatória.

O planejamento da alta é definido pela Organização Munidal da Sáude como uma estratégia de liberação do paciente de um centro especializado de cuidados para o domicílio ${ }^{(9)}$. Essa estratégia envolve um processo educativo que deve ocorrer em diferentes momentos do processo da internação a fim de promover maior segurança ao paciente e família, propiciar o esclarecimento de dúvidas, permitir a atualização de informações e assegurar a continuidade do cuidado no domicílio ${ }^{(10)}$. Ainda, nesse processo deverá haver envolvimento do paciente e família de maneira ativa a fim de encorajá-los a aceitarem responsabilidades, enfrentarem limitações e assumirem o controle da própria saúde ${ }^{(11)}$. Nesse sentido, pode-se dizer que o planejamento da alta visa a redução das barreiras e dificuldades percebidas no processo da doença e hospitalização, bem como para a realização das atividades do autocuidado ${ }^{(3)}$.

O autocuidado é definido pela realização das atividades práticas pelo indivíduo em seu próprio benefício para manutenção de sua saúde, bem-estar e desenvolvimento ${ }^{(12)}$. Esse fenômeno foi mencionado pela primeira vez na enfermagem por Orem ${ }^{(12)}$ e definido com base nas características dos indivíduos e no contexto em que se encontram de acordo com três sistemas: totalmente compensatório; parcialmente compensatório; e de apoio e educação. No sistema totalmente compensatório, o indivíduo é incapaz de realizar as atividades de autocuidado, com necessidade das intervenções do enfermeiro. No parcialmente compensatório, tanto o enfermeiro como o indivíduo executam as ações de cuidado; e, no sistema de apoio e educação, o indivíduo consegue executar as ações de autocuidado e aprende a realizá-las com o auxílio do enfermeiro que atua na função de educador ${ }^{(12)}$. Ademais, na área de enfermagem, o desenvolvimento do autocuidado ocorre por meio de acões de ensino, orientação e educação que visam a manutenção do processo de vida e funcionamento do ser humano. 
No entanto, frequentemente, os pacientes obtêm alta hospitalar e retornam ao domicílio ansiosos, inseguros e com dúvidas relacionadas ao autocuidado, incluindo aquelas relacionadas à re-educação alimentar, prática de exercícios físicos, retorno ao trabalho, sintomas esperados após a alta, prática de atividades diárias, dificuldades com as incisões cirúrgicas, prática de atividade sexual, consumo de bebida alcoólica e uso de medicações ${ }^{(13)}$. Mesmo frente a todos desafios do procedimento cirúrgico (dor, afastamento da família e dispositivos invasivos), os pacientes sentem-se mais seguros durante a internação, pois dispõem do suporte dos profissionais de saúde ${ }^{(14-15)}$. Ademais, frequentemente, a insegurança do paciente e cuidador no momento da alta se deve a limitações na prática do ensino de enfermagem, que geralmente ocorre de forma desestruturada e não sistematizada ${ }^{(15)}$.

Nesse sentido, destaca-se a necessidade de fortalecer o preparo da unidade família-indivíduo para o autocuidado no domićlio, permitindo que o autocuidar envolva menos dúvidas, sendo mais seguro e efetivo. Baseado nisso, a criação de uma ferramenta educativa pode ser considerada uma estratégia de apoio e educação a ser utilizada pelos enfermeiros para subsidiar o planejamento do autocuidado e de ações que orientem à alta hospitalar do paciente revascularizado. Acredita-se que, devido ao elevado número de indivíduos atingidos pela doença coronariana e que se submetem à cirurgia de revascularização do miocárdio (RM), o manual educativo seja uma potente ferramenta de ensino que auxilie paciente e família no autocuidado e ofereça maior segurança durante a alta hospitalar. Assim, espera-se menor risco de complicações, redução da taxa de retornos e reinternações e dos custos hospitalares a pacientes e instituições de saúde.

Todavia, embora já existam manuais voltados ao pós-operatório de cirurgias em geral ${ }^{(16)}$, a literatura atual é limitada em relação a manuais direcionados ao pós-operatório de revascularização miocárdica. Autores de pesquisa que evidenciou validade de um manual educativo para o autocuidado de mulheres mastectomizadas sugerem a construção de outros materiais didáticos ${ }^{(16)}$, pois a educação em saúde continua a ser objeto de interesse político, institucional e profissional visto seu potencial preventivo e para redução de custos com saúde ${ }^{(16)}$. Diante do exposto, o objetivo desse estudo foi elaborar um manual educativo para o autocuidado de paciente revascularizado e família após a alta hospitalar.

\section{MÉTODO}

Trata-se de uma pesquisa metodológica, cuja trajetória envolveu as seguintes fases:

1. Levantamento bibliográfico e de informações dos pacientes/familiares revascularizados e especialistas em cardiologia;

2. Estruturação da ferramenta educativa com base nos referenciais teóricos de Orem e de construção de instrumento de Pasquali;

3. Validação do conteúdo e de face da ferramenta educativa.

Na primeira fase, realizou-se o levantamento bibliográfico mediante a revisão integrativa de artigos publicados no portal PubMed. Foram utilizados os unitermos em inglês "coronary arterial bypass", "patient 
education as a topic", "patient discharge". Os artigos incluídos foram àqueles disponíveis na íntegra e nos idiomas português, inglês ou espanhol publicados entre o período de 2008 a 2012 e direcionados à pergunta: "Quais orientações educativas mais frequentes sobre autocuidado são abordadas no pós-operatório da cirurgia de revascularização miocárdica?". Foram excluídas as produções que utilizaram crianças e adolescentes como amostra e as pesquisas em animais. Assim, sete artigos foram selecionados e utilizados para guiar a construção do instrumento.

A partir dos temas de orientação hospitalar encontrados na literatura, foi construído um instrumento inicial com 46 itens e 10 categorias. Cada item foi avaliado pelos pacientes e, posteriormente, pelos peritos, sendo classificados segundo seu grau de importância para a realização de autocuidado, conforme segue:

1. Nada importante;

2. Pouco importante;

3. Muito importante.

Ao final, foram incluídas três perguntas abertas com o objetivo de coletar o maior número possível de informações dos pacientes/família e peritos. Nessa fase, a coleta de dados foi realizada entre fevereiro e abril de 2012 por meio de entrevista individualizada com duração aproximada de uma hora junto aos pacientes e/o familiares e de 30 minutos junto aos peritos. Para o tratamento das respostas, foi utilizada a análise de conteúdo de Bardin em três etapas: pré-análise, exploração do material e tratamento dos resultados e interpretação ${ }^{(17)}$.

$\mathrm{Na}$ segunda fase, procedeu-se à construção do manual educativo piloto com base no referencial metodológico de Pasquali. Assim, seguiram-se os critérios propostos: objetividade, simplicidade, clareza, relevância, precisão, variedade, modalidade, credibilidade, amplitude e equilíbrio ${ }^{(18)}$. Para inclusão dos itens, o nível de concordância dos pacientes/familiares deveria ser superior a 90\% e dos peritos igual ou superior a 75\% com a categoria "muito importante". Optou-se por trabalhar com essa porcentagem de concordância devido ao reduzido número de peritos e ao risco de se excluir itens pertinentes à construção do manual. Desta forma, os itens foram agrupados em 10 categorias:

1. Uso do medicamento;

2. Sinais e sintomas que você deve reconhecer;

3. Cuidados com a ferida operatória;

4. Alívio da dor;

5. Alimentação;

6. Utilização de bebidas alcoólicas e controle do tabagismo;

7. Alteração do padrão de sono;

8. Atividades;

9. Retorno ao trabalho;

10. Depressão e alterações emocionais.

Na terceira fase, ocorrida também no período de fevereiro a abril de 2012, realizou-se a avaliação 
semântica para se analisar a compreensão, relevância e adequação do conteúdo para a população em questão. As instruções e ilustrações também foram consideradas na avaliação do manual piloto. Dessa forma, foram avaliadas as ilustrações, aspectos teóricos (análise de conteúdo) e de linguagem (análise de face), sendo as categorias três e quatro avaliadas apenas quanto à linguagem e conteúdo e as demais quanto aos três aspectos. Para tais análises, foi elaborado um instrumento baseado no modelo utilizado por Luz e colaboradores $^{(19)}$, sendo adicionadas, no campo "conteúdo" do instrumento, questões para a análise objetiva do autocuidado em cada categoria. Por fim, o manual educativo piloto foi avaliado segundo seu formato, tamanho e apresentação, sendo possíveis comentários e sugestões.

Os sujeitos do estudo foram pacientes que se encontravam entre o terceiro e o oitavo dia pósoperatório de RM e/ou seus familiares, maiores de 18 anos, em condições de deambulação e de realização de tarefas mínimas de autocuidado, com capacidade cognitiva aparentemente preservada e alfabetizados ou analfabetos acompanhados por um dos familiares que soubesse ler e escrever. Entretanto, foram excluídos os pacientes com história clínica de RM prévia. A escolha dos participantes baseou-se na amostragem não probabilística intencional. Assim, a amostra da primeira fase (seleção dos itens para composição do manual educativo piloto) foi formada de 35 pacientes/familiares e da terceira fase (avaliação do manual educativo piloto) por oito pacientes/familiares.

O comitê de especialistas foi composto por oito profissionais com experiência em pós-operatório de cirurgia cardíaca, dentre eles: um cardiologista clínico, um cirurgião cardíaco, três enfermeiros, um fisioterapeuta, um nutricionista, um farmacêutico clínico e um psicólogo. O maior número de enfermeiros justifica-se pela importância desse profissional nas orientações para a alta hospitalar, sendo a seleção dos profissionais realizada com base no seu conhecimento e experiência prévia junto a pacientes cardiopatas. As diferentes categorias profissionais foram determinadas com o intuito de se obter um manual educativo abrangente e com orientações multiprofissionais.

A fim de se construir um manual atrativo e de fácil compreensão, incluíram-se ilustrações (fotos, desenhos e figuras) selecionadas a partir de páginas eletrônicas de acesso livre, pesquisadas no site "Google Images" (https://www.google.com.br/imghp?hl=pt-BR\&tab=wi\&authuser=0) e modificadas com o uso do software “Pages 2009" (versão 4.3/1048, Apple Inc., 2012). Após a coleta, os dados foram organizados em tabelas mediante o uso dos programas de computador Numbers 2009 e Microsoft Excel. Para as análises descritivas, foi utilizado o Statistical Package for Social Sciences (SPSS Versão 15).

A pesquisa atendeu as Normas da Resolução no 196/96 com parecer 85.122. Além disso, foi obtida autorização da Comissão de Ética para Análise de Projetos de Pesquisa (CAPPesq) do Hospital das Clínicas da Faculdade de Medicina da Universidade de São Paulo e da Coordenação de Enfermagem do Instituto do Coração da referida instituição. Cabe mencionar que os participantes que aceitaram participar da pesquisa formalizaram seu consentimento ao assinar o Termo de Consentimento Livre e Esclarecido. 


\section{RESULTADOS}

Na revisão de literatura, foram identificados 37 temas abordados na orientação de alta hospitalar dos pacientes submetidos à revascularização miocárdica (Tabela 1).

Tabela 1: Descrição dos temas encontrados e sua frequência na literatura. São Paulo, SP, Brasil, 2012.

\begin{tabular}{|c|c|c|c|c|c|}
\hline Item & Tema (Frequência) & $\%$ & Item & Tema (Frequência) & $\%$ \\
\hline 1 & Deambulação precoce & $62,5 \%$ & 20 & Utilização de medicamentos & $25 \%$ \\
\hline 2 & Controle da dor & $62,5 \%$ & 21 & Efeitos adversos aos medicamentos & $25 \%$ \\
\hline 3 & Oferta de suporte emocional & $62,5 \%$ & 22 & Pacientes com sentimento de vulnerabilidade & $25 \%$ \\
\hline 4 & Manutenção de condicionamento físico & $50 \%$ & 23 & Orientações quanto à tosse & $12,5 \%$ \\
\hline 5 & Realização de exercício físico & $50 \%$ & 24 & Restrição de líquidos & $12,5 \%$ \\
\hline 6 & Sintomas de tristeza e depressão & $50 \%$ & 25 & Alteração no padrão de sono & $12,5 \%$ \\
\hline 7 & Manejo da fadiga & $37,5 \%$ & 26 & Dor Abdominal & $12,5 \%$ \\
\hline 8 & Utilização de escadas & $37,5 \%$ & 27 & Controle do débito urinário & $12,5 \%$ \\
\hline 9 & Sinais de cicatrização da ferida operatória & $37,5 \%$ & 28 & Distensão abdominal & $12,5 \%$ \\
\hline 10 & Constipação & $37,5 \%$ & 29 & Diarreia & $12,5 \%$ \\
\hline 11 & Manejo da ansiedade & $37,5 \%$ & 30 & Retorno às consultas médicas & $12,5 \%$ \\
\hline 12 & Alteração do padrão de batimento cardíaco & $25 \%$ & 31 & Utilização de antiagregantes & $12,5 \%$ \\
\hline 13 & Orientações sobre respirações profundas & $25 \%$ & 32 & Utilização de hipolipemiantes & $12,5 \%$ \\
\hline 14 & Controle de peso/Perda de peso & $25 \%$ & 33 & Utilização de diuréticos & $12,5 \%$ \\
\hline 15 & Limpeza da ferida cirúrgica & $25 \%$ & 34 & Finalidade dos medicamentos & $12,5 \%$ \\
\hline 16 & $\begin{array}{l}\text { Sintomas de dor ou formigamento nos } \\
\text { membros superiores }\end{array}$ & $25 \%$ & 35 & Orientações quanto ao uso de meias elásticas & $12,5 \%$ \\
\hline 17 & Controle da náusea e inapetência & $25 \%$ & 36 & Inclusão da família nas orientações de alta & $12,5 \%$ \\
\hline 18 & Controle de exames laboratoriais & $25 \%$ & 37 & $\begin{array}{l}\text { Orientações sobre o retorno das atividades de } \\
\qquad \text { vida/ sociais/trabalho }\end{array}$ & $12,5 \%$ \\
\hline 19 & Uso de anticoagulantes & $25 \%$ & & & \\
\hline
\end{tabular}

A partir desses temas, foram propostos inicialmente 46 itens. Os pacientes/familiares classificaram 26 itens (57\%) como "muito importante", havendo entre 91,4\% e 100\% de concordância dos sujeitos para com essa classificação. Os itens que obtiveram $100 \%$ de concordância foram: Indicar o seguimento de dieta especial; Orientar os sinais e sintomas que devem ser observados caso presença de infecção da ferida operatória; Recomendar a limpeza e a segurança da casa; Indicar as ações que devem ser realizadas caso o paciente se sinta mal em casa; Explicar sobre os medicamentos que serão utilizados; Orientar sobre a interação medicamentosa; e Alertar os principais problemas encontrados com o uso dos medicamentos (efeitos colaterais).

Já no grupo de peritos, os profissionais determinaram 29 itens (63\%) como "muito importante", sendo que a concordância dos profissionais para com essa classificação foi igual ou superior a $75 \%$. Os itens que obtiveram 100\% de concordância foram: Orientar sobre o retorno à prática de esportes; Indicar o seguimento de dieta especial; Orientar sobre a ingestão de bebida alcoólica; Recomendar os cuidados com a ferida operatória; Orientar os sinais e sintomas que devem ser observados caso presença de infecção na ferida operatória; Indicar as ações que devem ser realizadas caso o paciente sinta mal em casa; Orientar sobre o retorno à consulta médica; Alertar sobre os riscos do fumo; e Recomendar alternativas para cessação do tabagismo.

Para construção do manual educativo piloto, associaram-se os dados obtidos pelas análises do grupo 
de pacientes/familiares e dos peritos. Assim, foram selecionados 36 itens para serem incorporados no manual educativo. Dos 36 itens, 19 (53\%) preencheram o critério de seleção para ambos os grupos, 10 (28\%) foram selecionados pelos peritos e sete (19\%) foram selecionados somente por pacientes.

Observa-se predomínio de $100 \%$ de concordância em todas categorias avaliadas pelos pacientes quanto ao conteúdo. Já a concordância dos pacientes quanto à linguagem e ilustrações da categoria "Sinais e Sintomas" foi de $87 \%$. Na avaliação dos peritos, houve $87,5 \%$ de concordância quanto ao conteúdo das categorias "Alimentação" e "Depressão e problemas emocionais"; 87,5\% de concordância quanto à linguagem das categorias "Uso de medicamentos", "Atividades" e "Retorno ao trabalho"; e 75\% quanto ao mesmo aspecto da categoria "Alimentação". Nas demais categorias, houve $100 \%$ de concordância quanto aos aspectos analisados. Considerando um mínimo de concordância de 75\%, todas as categorias avaliadas foram mantidas no manual. Assim, o Manual Educativo final compõem-se de 36 itens distribuídos em 26 categoriais, conforme Quadro 1.

Quadro 1: Itens da versão final do Manual Educativo para o autocuidado em pós-operatório de revascularização miocárdica. São Paulo, SP, Brasil, 2012.

\begin{tabular}{|c|}
\hline 1. Explicar a possibilidade de cortar, diluir, macerar os medicamentos \\
\hline 2. $\quad$ Explicar sobre os alimentos que devem ser evitados \\
\hline 3. Explicar sobre os medicamentos que serão utilizados \\
\hline 4. Indicar alternativas para reduzir os gastos com os medicamentos \\
\hline 5. Indicar as ações que devem ser realizadas caso o paciente se sinta mal em casa \\
\hline 6. Indicar o seguimento de dieta especial \\
\hline 7. Orientar a como realizar movimentos com auxílio (sentar no sofá, sair da cama) \\
\hline 8. $\quad$ Orientar a lidar com a ansiedade, como comunicar medos e preocupações \\
\hline 9. $\quad$ Orientar a utilização de insulina, caso haja necessidade \\
\hline 10. Orientar os sinais e sintomas que devem ser observados caso exista uma infecção na ferida operatória \\
\hline 11. Orientar sobre a adaptação psicológica \\
\hline 12. Orientar sobre a ingestão de bebida alcoólica \\
\hline 13. Orientar sobre a interação entre os medicamentos \\
\hline 14. Orientar sobre o retorno á consulta médica \\
\hline 15. Orientar sobre o retorno á prática de esportes \\
\hline 16. Orientar sobre o retorno da atividade sexual \\
\hline 17. Orientar sobre subir escadas \\
\hline 18. Recomendar a utilização de medicamentos genéricos \\
\hline 19. Recomendar os cuidados com a ferida operatória \\
\hline 20. Alertar sobre os riscos do fumo \\
\hline 21. Esclarecer quanto ao retorno da atividade de dirigir \\
\hline 22. Esclarecer sobre o retorno as atividades sociais (frequentar restaurantes, cinema, shopping) \\
\hline 23. Explicar estratégias para o paciente se vestir \\
\hline 24. Indicar a possibilidade de realizar viagem longa de avião \\
\hline 25. Orientar sobre as atividades de higiene pessoal \\
\hline 26. Orientar sobre da atividade de entrar no mar ou na piscina \\
\hline 27. Recomendar a utilização de meias elásticas \\
\hline 28. Recomendar alternativas para cessação do tabagismo \\
\hline 29. Relacionar as alterações emocionais com o pós-operatório \\
\hline 30. Alertar os principais problemas encontrados com o uso dos medicamentos (efeitos colaterais) \\
\hline 31. Explicar o mecanismo de ação de cada medicamento \\
\hline 32. Explicar quanto a exposição da ferida operatória ao sol \\
\hline 33. Explicar sobre a utilização de materiais de curativo (soro, gaze, luvas) em casa \\
\hline 34. Identificar como os medicamentos estão fazendo efeito \\
\hline 35. Informar o tempo de duração dos medicamentos (tempo de ação) \\
\hline 36. Recomendar a limpeza e a segurança da casa \\
\hline
\end{tabular}

A versão final, na íntegra, do Manual Educativo de cuidados no pós-operatório de revascularização 
miocárdica pode ser encontrado no link: https://marques-sm.wixsite.com/manualeducativopocrm.

\section{DISCUSSÃO}

O autocuidado é descrito como uma prática de comportamento desempenhada pelos indivíduos e fundamentada no propósito de manter e melhorar a qualidade de sua saúde ${ }^{(7)}$. Ela exige que os indivíduos possuam conhecimento sobre a sua condição de saúde, o que permite a eles assumirem o controle da mesma e a mudarem os comportamentos de risco ao optarem por hábitos de vida mais saudáveis ${ }^{(20)}$. Nesse contexto, destaca-se a educação como prática social, construída por intermédio da participação, do diálogo e dos significados atribuídos pelos indivíduos ${ }^{(19)}$, representada no manual desenvolvido nessa pesquisa por meio de sua estrutura, organização didática de conteúdo e desenho gráfico ${ }^{(20)}$.

A construção da ferramenta educativa foi baseada nas informações coletadas, não só dos profissionais da saúde, mas também da população-alvo, o que também foi utilizado em outros estudos ${ }^{(16,21)}$. Nesse sentido, em investigação ${ }^{(16)}$ cujo objetivo foi desenvolver um manual educativo direcionado à mulher mastectomizada, a validação realizada por um grupo de mulheres mastectomizadas e de juízes, especialistas no assunto. Em outro material educativo ${ }^{(21)}$, a promoção da saúde das gestantes foi fundamentada no levantamento das demandas do público-alvo e na revisão da literatura, assim como na sistematização do conteúdo e sua validação por peritos e gestantes. Alguns estudos ${ }^{(16,21)}$ reforçam que as demandas e manifestações levantadas entre os indivíduos, tanto em grupos selecionados, quanto em peritos no assunto, contribuem para a elaboração de materiais didáticos, pois retratam e auxiliam na construção teórica e voltam-se às necessidades reais percebidas pela população-alvo que vive a condição de saúde-doença.

Da mesma forma, a visão dos peritos é importante na elaboração de materiais de ensino e educação, pois contribui com conhecimentos específicos ao instrumento e informações pertinentes ao paciente. Outro benefício da construção de ferramentas com base na população-alvo é a maior tangibilidade do acesso à informação e ao aprendizado, facilitando a produção do conhecimento. Por isso, a elaboração dos materiais educativos em saúde, embora guiada pela expertise e experiência dos profissionais, deve fundamentar-se na valorização das demandas de conhecimento da população ${ }^{(16,21)}$. Com base nisso, destaca-se que a populaçãoalvo dessa pesquisa contribuiu efetivamente para a validade do conteúdo, de face e para a formatação da ferramenta educativa. Isso porque sua participação permitiu o fortalecimento e a abordagem de aspectos teóricos importantes no pós-operatório de cirurgia de RM, a apresentação do conteúdo do material de maneira organizada e atrativa, contribuindo para a efetiva leitura dos mesmos; e a coerência das informações apresentadas com aquelas realmente percebidas como importantes pelos respondentes.

Por outro lado, observa-se que, nos países em desenvolvimento, aproximadamente $50 \%$ da população adulta é analfabeta ${ }^{(22)}$, o que reforça a importância do envolvimento da família no processo de educação e da estruturação das ferramentas de ensino. Além disso, uma parcela da população apresenta algum grau de dificuldade para a leitura e compreensão das informações, sendo a família a unidade responsável por participar ativamente do cuidado no domicílio ${ }^{(23)}$. Portanto, esse manual foi construído considerando-se 
também a participação dos familiares que, em muitas ocasiões, são efetivos cuidadores do paciente no domicílio. Destaca-se que, muitas vezes, os profissionais envolvidos no cuidado hospitalar não conseguem contribuir com orientações focadas nas necessidades do paciente para o período pós-alta ${ }^{(22)}$. Nesse conexto, os familiares também podem se beneficiar de uma ferramenta que, estrategicamente, contemple as orientações pertinentes à alta e que sejam úteis na assistência ao cuidado do paciente no domicílio. Portanto, a inclusão dos familiares nesta pesquisa também contribuiu para a validade do conteúdo e semântica, bem como para a formatação e construção da ferramenta educativa. Sua participação contribuiu para que o material final fosse de fácil compreensão e, ao mesmo tempo, constituído por informações que o mesmo considerava como necessárias para o cuidado. Assim, espera-se que o familiar consiga interpretar e utilizar as informações do manual para o auxílio ao paciente em pós-operatorio de cirurgia de RM.

O material escrito facilita o processo de educação, pois permite que o leitor supere as dificuldades de compreensão por meio da decodificação de informações e rememorização do conteúdo ${ }^{(23-24)}$. Isso foi verificado em revisão sistemática junto a pacientes sob tratamento medicamentoso ${ }^{(24)}$, na qual se evidenciou que pacientes e familiares preferem informações escritas para auxiliá-los na tomada de decisão no domicílio. No entanto, embora a comunicação escrita seja uma ferramenta indispensável à divulgação de boas práticas em saúde, existem aspectos relacionados à linguagem, ilustrações e layout que devem ser considerados para a elaboração dos materiais educativos impressos a fim de torná-los facilmente compreensíveis, legíveis e relevantes ${ }^{(23-24)}$. Assim, são preferíveis materiais com ilustrações coloridas do que bicolores (preto e branco), pois estas últimas não estimulan a atenção e o interesse do leitor ${ }^{(21-22)}$. Assim, sugere-se que os materiais impressos, confeccionados para chamarem a atenção da população-alvo, devem ser, informativos e persuasivos para a mudança de comportamento, retratando temas de impacto à saúde. Portanto, a clareza da linguagem torna-se fundamental para a divulgação da informação, levando-se em consideração que, nos materiais escritos, o leitor está sozinho frente à interpretação. Assim, as linguagens técnico-científicas, ambíguas e exclusivamente populares atrapalham a interpretação das informações e contribuem para o surgimento de dúvidas ${ }^{(15)}$.

Incluíram-se recursos adicionais de apoio psicológico e suporte social (endereços eletrônicos, contato telefônico e serviços de saúde referenciados) na ferramenta educativa impressa, como forma de propiciar a busca de novas informações e, assim, auxiliar ainda mais os pacientes e familiares na compreensão e no desempenho do autocuidado ${ }^{(7-8)}$. Sobre isso, destaca-se que a utilização de recursos educativos para o autocuidado está associada a níveis elevados de conhecimento para a identificação de problemas de saúde, ao auto-atendimento e ao desempenho de ações comportamentais que reduzem o aparecimento de sintomas físicos e emocionais ${ }^{(15)}$. Portanto, desenvolver a capacidade do indivíduo e sua família nestas habilidades, no pós-operatório da cirurgia de RM, contribui para o aumento da qualidade de vida e diminui a incidência de complicações cardiovasculares secundárias. 


\section{CONCLUSÃO}

A construção e validação de conteúdo e semântica desta ferramenta focada em uma amostra específica da população foi considerada satisfatória. Ela pode ser utilizada para otimizar o planejamento da alta pelos profissionais da saúde e para auxiliar pacientes e familiares no cuidado pós-alta hospitalar no domicilio. Isso reduzirá as taxas de retorno de pacientes com complicações clínicas decorrentes do uso incorreto de medicações, manuseio da ferida operatória e do desconhecimento do processo de reabilitação de forma geral. Portanto, a aplicação desse instrumento, na prática, permitirá um pós-operatório de revascularização miocárdica mais seguro e com menor risco de complicações no domicílio decorrentes do despreparo para o autocuidado, reduzindo o número de reinternações e os custos hospitalares, além de padronizar o ensino de enfermagem.

Além disso, o pós-operatório, quando orientado para a alta de forma específica e fundamentado nas características de uma determinada população, permite maior aproximação entre paciente/família e autocuidado. Isso reduz as barreiras da orientação empírica e despersonalizada, fortalece o foco em estratégias interessantes para a unidade paciente-família e aumenta a segurança, praticidade e confiança na execução do cuidado.

Como limitação, aponta-se sua especificidade aos pacientes em pós-operatório de cirurgia de revascularização miocárdica, não sendo aplicável a pacientes submetidos a outros procedimentos cirúgicos existentes. Por isso, sugere-se que novas ferramentas sejam desenvolvidas para outras populações que demandam cuidados pós-operatórios no domicílio.

\section{REFERÊNCIAS}

1. World Health Organization. The top 10 causes of death. The 10 leading causes of death in the world, 2000 and 2012 [Internet]. World Health Organization; 2014 [citado 2015 nov. 25]. Disponível em:

http://www.who.int/mediacentre/factsheets/fs310/en/

2. Goss F, Brachmann J, Hamm CW, Haerer W, Reifart N, Levenson B. High adherence to therapy and low cardiac mortality and morbidity in patients after acute coronary syndrome systematically managed by office-based cardiologists in Germany: 1-year outcomes of the ProAcor Study. Vasc Health Risk Manag. 2017;13:127-37.

3. Brown JP, Clark AM, Dalal H, Welch K, Taylor RS. Patient education in the management of coronary heart disease. Cochrane Database Syst Rev. 2011;(12): CD008895.

4. Polanczyk CA, Ribeiro JP. Coronary artery disease in Brazil: contemporary management and future perspectives. Heart. 2009;95(11):870-6.

5. Muller LA, Rabelo ER, Moraes MA, Azzolin K. Delay factors on the administration of thrombolytic therapy in patientes with acute myocardial infarction in a general hospital. Rev Latinoam Enferm. 2008; 16(1):52-6.

6. Dordetto PR, Pinto GC, Rosa TCSC. Pacientes submetidos à cirurgia cardíaca: caracterização sociodemográfica, perfil clínico-epidemiológico e complicações. Rev Fac Ciênc Méd Sorocaba. 2016;18(3):144-9.

7. Fredericks S, Sidani S. An exploration of the relationship between coronary artery bypass graft patients' self-sought educational resources and outcomes. J Cardiovasc Nurs. 2008;23(5):422-31.

8. Mistiaen P, Francke AL, Poot E. Interventions aimed at reducing problems in adult patients discharged from hospital to home: a systematic meta-review. BMC Health Serv Res. 2007; 7(47):1-19.

9. Huber DL, McClelland E. Patient preferences and discharge planning transitions. J Prof Nurs. 2003;19(4):204-10. 
10. The Joint Comission. The Joint Comission Guide to Patient and Family Education. Oakbrook Terrace: The Joint Commission; 2010.

11. Folkman S, Lazarus RS. Stress, Appraisal, and Coping. New York: Springer Publishing Company; 1984.

12. Santos I, Sarat CNF. Modalidades de aplicação da Teoria do Autocuidado de Orem em comunicações científicas de enfermagem brasileira. Rev Enferm UERJ. 2008;16(3):313-8.

13.Almeida PFP, Júnior RG, Gasparino RC. Dúvidas dos pacientes em pós-operatório de Revascularização do miocárdio*. Cogitare Enferm. 2009; 14(4):675-81.

14. Erdmann AL, Lanzoni GMM, Callegaro GD, Baggio MA, Koerich C. Compreendendo o processo de viver significado por pacientes submetidos a cirurgia de revascularização do miocárdio. Rev Latinoam Enferm. 2013; 21(1):1-8.

15. Castro AP, Oikawa SE, Domingues TAM, Hortense FTP, Domenico EBL. Educação em Saúde na Atenção ao Paciente Traqueostomizado: Percepção de Profissionais de Enfermagem e Cuidadores. Rev bras cancerol. 2014; 60(4): 305-13. 16. Oliveira MS, Fernandes AFC, Sawada NO. Manual educativo para o autocuidado da mulher mastectomizada: um estudo de validação. Texto \& Contexto Enferm. 2008;17(1):115-23.

17. Bardin L. Análise de conteúdo. Lisboa: Edições 70; 2009.

18. Pasquali L. Psicometria: Teoria dos testes na Psicologia e na Educação. Petrópolis: Vozes; 2003.

19. Da Luz ZM, Pimenta DN, Rabello A, Schall V. Evaluation of informative materials on leishmaniasis distributed in Brazil: criteria and basis for the production and improvement of health education materials. Cad Saúde Pública. 2003;19(2):561-9.

20. Barrio-Cantalejo, Simón-Lorda P, Jiménez MM, Ruiz AM. Consensus on the legibility criteria of health education leaflets. An Sist Sanit Navar. 2011;34(2):153-65.

21. Reberte LM, Hoga LAK, Gomes ALZ. O processo de construção de material educativo para a promoção da saúde da gestante. Rev Latinoam Enferm. 2012; 20(1):101-8.

22. Moreira MF, Nobrega MM, Silva MIT. Comunicação escrita: contribuição para a elaboração de material educativo em saúde. Rev Bras Enferm. 2003; 56 (2):184-8.

23. McGlone MS, Bell RA, Zaitchik ST, McGlynn J. Don't let the flu catch you: agency assignment in printed educational materials about the H1N1 influenza virus. J Health Commun. 2013;18(6):740-56.

24. Armindo GL, Diniz MCP, Schall VT. Printed educational materials about dengue: qualitative and quantitative analysis and reflections about health communication and health education. Associação Brasileira de Pesquisa e Educação em Ciências (ABAPEC); 2011. 